\title{
Front Matter: Volume 11137
}

, "Front Matter: Volume 11137," Proc. SPIE 11137, Applications of Digital Image Processing XLII, 1113701 (14 November 2019); doi:

$10.1117 / 12.2551809$

SPIE Event: SPIE Optical Engineering + Applications, 2019, San Diego, California, SPIE. United States 


\title{
PROCEEDINGS OF SPIE
}

\section{Applications of Digital Image Processing XLII}

\author{
Andrew G. Tescher \\ Touradj Ebrahimi \\ Editors
}

12-15 August 2019

San Diego, California, United States

Sponsored and Published by

SPIE 
The papers in this volume were part of the technical conference cited on the cover and title page. Papers were selected and subject to review by the editors and conference program committee. Some conference presentations may not be available for publication. Additional papers and presentation recordings may be available online in the SPIE Digital Library at SPIEDigitallibrary.org.

The papers reflect the work and thoughts of the authors and are published herein as submitted. The publisher is not responsible for the validity of the information or for any outcomes resulting from reliance thereon.

Please use the following format to cite material from these proceedings:

Author(s), "Title of Paper," in Applications of Digital Image Processing XLII, edited by Andrew G. Tescher, Touradj Ebrahimi, Proceedings of SPIE Vol. 11137 (SPIE, Bellingham, WA, 2019) Seven-digit Article CID Number.

ISSN: 0277-786X

ISSN: 1996-756X (electronic)

ISBN: 9781510629677

ISBN: 9781510629684 (electronic)

Published by

SPIE

P.O. Box 10, Bellingham, Washington 98227-0010 USA

Telephone +13606763290 (Pacific Time) · Fax +1 3606471445

SPIE.org

Copyright @ 2019, Society of Photo-Optical Instrumentation Engineers.

Copying of material in this book for internal or personal use, or for the internal or personal use of specific clients, beyond the fair use provisions granted by the U.S. Copyright Law is authorized by SPIE subject to payment of copying fees. The Transactional Reporting Service base fee for this volume is $\$ 21.00$ per article (or portion thereof), which should be paid directly to the Copyright Clearance Center (CCC), 222 Rosewood Drive, Danvers, MA 01923. Payment may also be made electronically through CCC Online at copyright.com. Other copying for republication, resale, advertising or promotion, or any form of systematic or multiple reproduction of any material in this book is prohibited except with permission in writing from the publisher. The CCC fee code is 0277$786 \times / 19 / \$ 21.00$.

Printed in the United States of America by Curran Associates, Inc., under license from SPIE.

Publication of record for individual papers is online in the SPIE Digital Library.

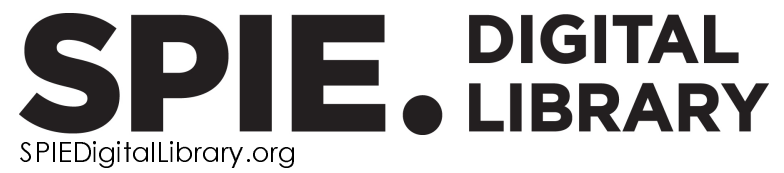

Paper Numbering: Proceedings of SPIE follow an e-First publication model. A unique citation identifier (CID) number is assigned to each article at the time of publication. Utilization of CIDs allows articles to be fully citable as soon as they are published online, and connects the same identifier to all online and print versions of the publication. SPIE uses a seven-digit CID article numbering system structured as follows:

- The first five digits correspond to the SPIE volume number.

- The last two digits indicate publication order within the volume using a Base 36 numbering system employing both numerals and letters. These two-number sets start with $00,01,02,03,04$, 05, 06, 07, 08, 09, OA, OB ... 0Z, followed by 10-1Z, 20-2Z, etc. The CID Number appears on each page of the manuscript. 


\title{
Contents
}

\author{
ix Authors \\ xiii Conference Committee
}

MEDICAL IMAGING

1113702 Transfer learning for early detection and classification of amblyopia [11137-1]

1113704 Projection based subcutaneous vein detection imaging modality and its feasibility evaluation in optical phantom and human: a preliminary study [1 $11137-3]$

1113705 Motion robust imaging photoplethysmography in defocus blurring [11137-4]

1113706 Real-time and robust heart rate measurement for multi-people in motion using IPPG [11137-5]

MEDICAL IMAGING AND MICROSCOPY

1113707 Improve methodology for tumor detection in mammogram images [1 $1137-6]$

1113708 Evaluating resolution in live cell structured illumination microscopy [11137-7]

1113709 Comparative analysis of smoothing filters in confocal microscopy images [1 $1137-8]$

REMOTE SENSING AND SPACE IMAGING I

$11137 \mathrm{OB}$ Classification of ground objects from remote sensing image with close spectral curves based on modified density mixture model [1 $1137-10]$

11137 OD A jigger ship's automatic detection method based on VIIRS DNB data [11137-12]

REMOTE SENSING AND SPACE IMAGING II

$111370 G$ Comparative analysis of interest points detection techniques for the registration of infrared and visible aerial photographs in pasture crops [1 $1137-16]$

$11137 \mathrm{OH}$ Exploiting camera rolling shutter to detect high frequency signals [11137-17]

11137 Ol Identification of breakwater damage by processing video with the SURF algorithm [11137-18] 


\section{NEXT-GENERATION IMAGE COMPRESSION}

11137 OJ Parallelization and multi-threaded latency constrained parallel coding of JPEG XS [11137-19]

11137 OK JPEG XL next-generation image compression architecture and coding tools [11137-20]

11137 OL Rust AV1 encoder (rav1e) project [11137-21]

11137 OM A new end-to-end image compression system based on convolutional neural networks [1 $11137-22]$

11137 ON Assessment of quality of JPEG XL proposals based on subjective methodologies and objective metrics [1 $11137-23]$

PERCEPTUAL CODING, QUALITY OPTIMIZATIONS, AND ASSESSMENTS I

$111370 Q$ Content-adaptive frame level rate control for video encoding using a perceptual video quality measure [1 $11137-26]$

11137 OR Overnight large-scale subjective video quality assessment using automatic test generation and crowdsourcing Internet marketplaces [1 $11137-27]$

PERCEPTUAL CODING, QUALITY OPTIMIZATIONS, AND ASSESSMENTS II

11137 OS A NR-IQA based deep neural network for tone mapping HDR images [1 1137-28]

11137 OT Deep learning and video quality analysis [1 $1137-29]$

\section{IMAGE RESTORATION AND ENHANCEMENT I}

11137 OV Flicker reduction method for $120 \mathrm{fps}$ shooting under $100 \mathrm{~Hz}$ light fluctuation by using a double rolling shutter [1 $11137-31]$

11137 OW Blur and noisy image restoration for near real time applications [1 1137-32]

$11137 \mathrm{OX}$ Investigation of moving objects through atmospheric turbulence from a non-stationary platform [1 $11137-33]$

\section{FUTURE VIDEO CODING I}

$111370 Z$ Propagation of quantization error in performing intra-prediction with deep learning [11137-35]

iv 
1113710 MPEG-5: essential video coding standard [11137-36]

1113711 Hadamard transform domain filter for video coding [1 $1137-37]$

$1113712 \quad$ Noise suppression filter for video coding [11137-38]

1113713 Data Adaptive HDR compression in VVC [1 $1137-39]$

1113714 Transform skip residual coding for the versatile video coding standard [11137-40]

\section{FUTURE VIDEO CODING II}

1113715 Performance comparison of VVC, AV1 and EVC [11137-41]

1113716 Intra prediction using multiple reference lines for the versatile video coding standard [11137-42]

1113717 Perceptually-inspired super-resolution of compressed videos [11137-43]

1113718 AV1 in-loop super-resolution framework [11137-44]

LIGHT FIELD, POINT CLOUD, AND HOLOGRAPHIC IMAGING I

1113719 Point cloud compression on the basis of 3D motion estimation and compensation [11137-45]

11137 1A A method of level of details control table for 3D point density scalability in video based point cloud compression [1 $11137-46]$

11137 1B 3D map generation based on keyframes selection and keypoints tracking [11137-47]

11137 1C Per-pixel calibration using CALTag and dense 3D point cloud reconstruction [11137-48]

LIGHT FIELD, POINT CLOUD, AND HOLOGRAPHIC IMAGING II

11137 1D An exploratory study towards objective quality evaluation of digital hologram coding tools [1 $11137-49]$

$111371 \mathrm{E}$ Towards practical hologram streaming using progressive coding [11137-50]

11137 IF Overview of MV-HEVC prediction structures for light field video [11137-51]

$111371 G$ JPEG Pleno light field coding technologies [11137-52] 
$111371 \mathrm{H}$ Performance analysis of JPEG Pleno light field coding [1 1137-53]

$1113711 \quad$ Rendering-dependent compression and quality evaluation for light field contents [11137-54]

IMAGE RESTORATION AND ENHANCEMENT II

11137 1J Gaussian noise estimation methods in images [11137-55]

$111371 \mathrm{~K} \quad$ Accurate image dehazing with three simultaneously captured hazed images [1 $1137-56]$

$111371 \mathrm{~L} \quad$ Restoration of depth-based space-variant blurred images [1 $11137-58$ ]

IMAGE ANALYSIS AND SECURITY I

1113710 Banknotes classification system through image processing and pattern recognition for people with visual impairment [1 $11137-62]$

IMAGE ANALYSIS AND SECURITY II

11137 1P Hass avocado classification by color and volume using a Kinect sensor [1 $1137-63]$

$111371 Q$ The impact of message quality on entity location and identification performance in distributed situational awareness [1 $11137-64]$

$111371 R \quad$ Energy based image steganography using dynamic programming [11137-65]

$111371 \mathrm{~T} \quad$ Real-time multi-criteria classification of facial images [1 1137-67]

$111371 \mathrm{U}$ Spatial domain analysis based on human position and movement recognition for the top-view imaging systems [1 $11137-101]$

\section{POSTER SESSION}

11137 IW Product detection based on CNN and transfer learning [1 $1137-69]$

$111371 \mathrm{X}$ Colorimetric index-based segmentation for RGB images of whales [1 $1137-70]$

$111371 Y$ 3D face recognition using depth filtering and deep convolutional neural network [11137-71]

$1113712 \quad$ Neural eye-processing computer based on FPGA technologies [11137-72]

vi 
1113724 Classification of breast abnormalities in digital mammography using phase-based features [1 11 137-77]

1113725 Reconstruction of 3D deformable objects using a single Kinect sensor [1 $1137-78]$

1113726 Adaptive algorithm for the SLAM design with a RGB-D camera [1 $1137-79]$

1113728 Optimization of installation of deformation monitoring of multiple points by optical methods [1 $11137-82]$

$111372 \mathrm{~A}$ Individual identification automation in Crocodylians through imagery processing: American crocodile as a study case [11137-84]

$111372 \mathrm{~B} \quad$ New automatic method for tracking rats in a pool for medication studies [11137-85]

11137 2C Mathematical improvement of the Lee's 3D skeleton algorithm [11137-86]

$111372 \mathrm{E}$ Analysis of the convolutional neural network architectures in image classification problems [1 $1137-88]$

$111372 \mathrm{~F} \quad$ Extraction of phase profile at discrete spatial frequency bands using phase shifting interferometry [1 $11137-89]$

$111372 \mathrm{G}$ Stationarity testing in 2D image analysis [1 $1137-90]$

$111372 \mathrm{H} \quad$ Variational approach to semi-automated 2D image segmentation [1 $1137-91$ ]

$111372 \mathrm{~J} \quad$ Semantic segmentation approach for tunnel roads' analysis [11137-93]

$111372 \mathrm{~K} \quad$ Non-rigid ICP and 3D models for face recognition [11137-94]

$111372 \mathrm{~L} \quad$ An efficient 3D mapping framework [11137-95]

$111372 \mathrm{M}$ Image dehazing using spatially displaced sensors [11137-96]

$111372 \mathrm{~N}$ Index-based methods for water body extraction in satellite data [11137-97]

1113720 Thyroid nodule diagnosis system based on the densely connected convolutional network [1 $1137-98]$

$111372 \mathrm{P} \quad$ A compact representation of character skeleton using skeletal line based shape descriptor [1 $1137-99]$ 
Proc. of SPIE Vol. $111371113701-8$

Downloaded From: https://www.spiedigitallibrary.org/conference-proceedings-of-spie on 26 Apr 2023 Terms of Use: https://www.spiedigitallibrary.org/terms-of-use 


\section{Authors}

Numbers in the index correspond to the last two digits of the seven-digit citation identifier (CID) article numbering system used in Proceedings of SPIE. The first five digits reflect the volume number. Base 36 numbering is employed for the last two digits and indicates the order of articles within the volume. Numbers start with 00, 01, 02, 03, 04, 05, 06, 07, 08, 09, OA, OB...0Z, followed by 10-12, 20-2Z, etc.

Abul, Hasnat, 2J

Acosta, Andrea, $0 \mathrm{G}$

Afonso, Mariana, 17

Agah, Arvin, 02

Aguilar, Luis A., 2B

Akyazi, Pinar, OM, ON

Alakuijala, Jyrki, OK

Alvarez-Xochihua, Omar, 1B

Arce, Gonzalo R., 1C

Arreola-Esquivel, M., 2N

Astola, Pekka, 1G, $1 \mathrm{H}$

Avramelos, Vasileios, $1 \mathrm{~F}$

Balaguera-Reina, Sergio A., 2A

Balasubramanian, R., OW

Baquedano, Laura E., 2B

Barbato, Luca, OL

Barr, David M., OL

Bates, Chad T., 1Q

Bezkrevnyi, Oleksandr S., 1 Z

Bhakat, Sarad Subhra, $2 \mathrm{~F}$

Bhattacharya, Kallol, 2F

Birman, Raz, OZ

Bosch, Marc, 02

Boukortt, Sami, OK

Bross, Benjamin, 14, 16

Bruse, Martin, OK

Bull, David R., 17

Cadena, Luis, 07

Cagnazzo, Marco, 1E

Carmel, Sharon, $0 Q, 0 R$

Carvajal-Gámez, Blanca Esther, 1X

Castillo-Martínez, Miguel Ángel, 1X

Chakraborty, Ipsita, $2 F$

Chang, Yao-Jen, 16

Chao, Ming-Te, 2P

Chen, Chi, 20

Chen, Fei, 05

Chen, J., 10, 11, 12

Chen, Yue, 18

Chen, Yung-Sheng, 2O, 2P

Chen, Zhifeng, $1 \mathrm{~T}$

Chernyak, R., 10, 11, 12

Chien, W.-J., 10

Choi, K., 10

Choi, Kwang Pyo, OS, 10

Choi, Minseok, OS

Cibis, Gerhard W., 02

Comșa, Iulia-Maria, OK

Corda, Roberto, 1D
Croitoru, Arie, $1 Q$

Crooks, Andrew, $1 Q$

Dai, Wei, OT, 13, 15

da Silva, Eduardo A. B., 1G, $1 \mathrm{H}$

David-Malka, Avishay, $\mathrm{OZ}$

Delgadillo-Herrera, M., 2N

Deng, Qifan, 06

Diaz-Escobar, Julia, 24

Dong, Liquan, 05, 06, 1W

Dorofeev, Konstantin, IY

Ducuara, Alvaro, OG

Ebrahimi, Touradj, OM, ON, 11

Egge, Nathan E., OL

El Khattabi, Yassine, 2J

El Rhammad, Anas, 1E

Ferrante, Nicholas, OX

Firsching, Moritz, OK

Fischbacher, Thomas, OK

Fliegel, Karel, 08, 2G, 2H

Forero, Manuel G., 09, 0G, 1J, 1O, 1P, 2A, 2B, 2C

Fößel, Siegfried, OJ

Franklin, Lena, $\mathrm{OH}$

Fujii, Toshiaki, 11

Funatsu, Ryohei, OV

Gallegos-Funes, Francisco Javier, 1X

Gao, C. X., OD

Ghosh, Payel, 2F

Gifford, Christopher M., 02

Gill, Dror, OQ, OR

Gilles, Antonin, 1D, 1E

Gilles, Jérôme, OX

Gioia, Patrick, 1E

Godoy, Yesmar, OG

Gómez A., Felipe, 1P

Gomez, Sebastian, OK

Gonzalez-Fraga, Jose A., 1B

Graham, Lior, 1L

Grange, Adrian, 18

Gyandendra, ., OW

Hadar, Ofer, OZ, $1 \mathrm{R}$

Han, Baoling, 06

Harclerode, Eric, 1Q

Harvie, David P., 02

Hernandez, Carlos A., OI

Hernandez, Natalia C., 2B

Herrera-Charles, Roberto, 01

Hu, Jian, OD

Huber, David, $\mathrm{OH}$

Hui, Mei, 05, 06, 1W 
Ikonin, S., 10, 11, 12

Im, Jiheon, 19, 1A

Im, Jinhyuck, 04

Jhu, Hong-Jheng, 16

Jian, Hui-Yu, 16

Joshi, Urvang, 18

Julius, Dan, OR

Jung, Byungjo, 04

Karczewicz, M., 10

Kaushik, Brajesh Kumar, OW

Keinert, Joachim, OJ

Keydel, Paul, 16

Khalil, Alami, 2J

Khanmohammad, Hesam, $1 \mathrm{H}$

Khata, Marouan, 2J

Kikuchi, Kodai, OV

Kim, Byeong Hak, $1 \mathrm{U}$

Kim, Junsik, 19, 1A

Kim, Kyuheon, 19, 1A

Kim, Min Young, $1 \mathrm{U}$

Kim, Young Hyoung, $1 \mathrm{U}$

Kitamura, Kazuya, OV

Klíma, Miloš, 08

Kliuchnikov, Evgenii, OK

Kober, Anastasia, $1 Y$

Kober, Olga, 1K

Kober, Vitaly, 1B, 1K, 1Y, 24, 25, 26, 2E, 2K, 2L, 2M

Kong, Lingqin, 05, 06, 1W

Kozhemyako, Andriy V., $1 \mathrm{Z}$

Krbcová, Zuzana, 2G, 2H

Krishnan, Madhu, OT, 13, 15

Kukal, Jaromír, 2G, 2H

Kumar, Rahul, OW

Kusakabe, Yuichi, OV

Lambert, Peter, $1 \mathrm{~F}$

Lau, Daniel L., $1 \mathrm{C}$

Lee, Seung Jun, $1 \mathrm{U}$

Leonov, Sergey, 2E

Li, Chuanrong, OD

Li, Cuiling, 05

Li, Xiang, 16

Liao, Li-Jen, 20

Lin, Min-Hsiung, 20

Liu, Bangan, 28

Liu, Ming, 05, 06, 1W

Liu, Shan, 16

Liv, Xiaohua, 05

Liu, Yaokai, OD

Llanza, Arcadi, 2J

López-Martínez, José Luis, $1 \mathrm{~K}$

Lozano, Juan J., 2A

$\mathrm{Ma}, \mathrm{Di}, 17$

Ma, Xiao, $1 \mathrm{C}$

Maarek, Paul, 2J

Makovetskii, Artyom, 2E, 2K, 2L, 2M

Marinov, Radoslav, $1 \mathrm{~T}$

Marpe, Detlev, 14, 16

Matsubara, Tomoki, OV

Meicler, Antoine, 2J
Miranda, Sergio L., 1J

Molina, Fabián, 2C

Molodetskikh, Ivan, OL

Montgomery, Christopher (Monty), OL

Morales, Erick G., Ol

Moreno H., Gustavo Andres, 10, 1P

Moreno, Juan Guillermo, 0G

Morera, Cristian M., 2B

Mukherjee, Debargha, 18

Murillo, Camilo, 2C

Nachtigalová, Iva, 2G, 2H

Nair, Tejas, OS

Nakib, Amir, 2J

Nguyen, Tung, 14

Noslier, Justine, 2J

Obryk, Robert, OK

Oh, Kwan-Jung, 1D

Ortiz-González, Antonio, 26

Padilla-Vivanco, A., 2N

Pagliari, Carla, 1G, $1 \mathrm{H}$

Parameswaran, Shibin, OX

Park, J., 10

Park, Jihoon, 04

Park, M. W., 10

Park, Pilkyu, OS

Parker, Sarah, 18

Peña-Ambrosio, Reynel, 09

Pena-Pena, Karelia, $1 \mathrm{C}$

Perra, Cristian, 1D, 1G, 1H

Pinheiro, Antonio, 1D

Pospísil, Jakub, 08

Potempa, Krzysztof, OK

Qian, Yonggang, OD

Qiu, Shi, OD

Ramírez N., Mauricio, $1 \mathrm{P}$

Ramos-Arredondo, Rosa Isela, 1X

Restrepo-Taborda, Camilo, 09

Reznik, Yuriy, $1 T$

Rhatushnyak, Alexander, OK

Rhyu, Sungryeul, 19, 1A

Richter, Thomas, OJ

Rojas-Rodríguez, Diana, 09

Ruchay, Alexey, 1Y

Ruiz-Rodriguez, Marcelo Luis, 25

Rusanovskyy, D., 10

S. P., Shreevari, OL

Sánchez-Tarquino, Jaime, 09

Sanogo, Assan, 2J

Saptsin, Vladimir, $1 \mathrm{~K}$

Sarkar, Sanjukta, 2F

Schelkens, Peter, 1D, 1G, 1H

Schwarz, Heiko, 14, 16

Segal, Yoram, $\mathrm{OZ}$

Seregin, $V_{\text {., }} 10$

Shmueli, Ron, OZ, 1R

Shmueli, Tal, $1 R$

Shoham, Tamar, OQ, OR

Shvai, Nadiya, 2J

Sneyers, Jon, OK 
Stepin, V., 11, 12

Su, Hui, 18

Švihlík, Jan, 08, 2G, 2H

Szabadka, Zoltan, OK

Tabus, loan, 1G, $1 \mathrm{H}$

Takahashi, Keita, 1 I

Tamayo Z., Kelly, 10

Terterov, Nikolay, $\mathrm{OQ}$

Tihonkih, Dmitrii, 2L

Tiktov, Pavel, $\mathrm{OQ}$

Tomioka, Kohei, OV

Topiwala, Pankaj, 0T, 13, 15

Toxqui-Quitl, C., 2N

van Asseldonk, Ruud, OK

Vandervenne, Lode, OK

Van Wallendael, Glenn, $1 \mathrm{~F}$

Vasilyev, Alexander, 2E

Vergara, Miguel A., $\mathrm{Ol}$

Versari, Luca, OK

Viola, Irene, 11

Voronin, Aleksei, 2K, 2L, 2M

Voronin, Sergei, 2K, $2 M$

Wang, Qi, OD

Wang, Weijie, 05, 06

Wang, Y.-K., 10

Wassenberg, Jan, OK

Watanabe, Osamu, $1 G$

Wei, Yongbin, 28

Wiegand, Thomas, 14, 16

Wu, Yuheng, 05, 06

Xue, Chengcheng, OD

Yasue, Toshio, OV

Yitzhaky, Yitzhak, $1 \mathrm{~L}$

Yu, Hyunseon, 04

Zhang, Fan, 17

Zhang, Ye, OB

Zhao, Liang, 16

Zhao, Xin, 16

Zhao, Yuejin, 05, 06, 1W

Zhou, Xinyu, OB

Zhu, Xingsheng, IW

Zuleta, Ricardo, 2C

Zumer, Raphaël A., OL 
Proc. of SPIE Vol. 11137 1113701-12

Downloaded From: https://www.spiedigitallibrary.org/conference-proceedings-of-spie on 26 Apr 2023 Terms of Use: https://www.spiedigitallibrary.org/terms-of-use 


\section{Conference Committee}

Program Track Chair

Khan M. Iftekharuddin, Old Dominion University (United States)

Conference Chairs

Andrew G. Tescher, AGT Associates (United States)

Touradj Ebrahimi, Ecole Polytechnique Fédérale de Lausanne

(Switzerland)

Conference Program Committee

Anne Margot Aaron, Netflix, Inc. (United States)

Vasudev Bhaskaran, Qualcomm Inc. (United States)

Antonin Descampe, intoPIX s.a. (Belgium)

Frederic Dufaux, CNRS (France)

Dan Grois, Comcast Corporation (Israel)

Ofer Hadar, Ben-Gurion University of the Negev (Israel)

Arianne T. Hinds, CableLabs (United States)

loannis Katsavounidis, Facebook Inc. (United States)

C.-C. Jay Kuo, The University of Southern California (United States)

Ajay Luthra, Picsel Laboratories (United States)

Andre J. Oosterlinck, KU Leuven Research and Development (Belgium)

Sethuraman Panchanathan, Arizona State University (United States)

Fernando Pereira, Instituto de Telecomunicações (Portugal)

Yuriy A. Reznik, Brightcove, Inc. (United States)

Thomas Richter, Fraunhofer-Institut für Integrierte Schaltungen (Germany)

John A. Saghri, California Polytechnic State University, San Luis Obispo (United States)

Peter Schelkens, Vrije Universiteit Brussel (Belgium)

Gary J. Sullivan, Microsoft Corporation (United States)

David S. Taubman, The University of New South Wales (Australia)

Pankaj Topiwala, FastVDO Inc. (United States)

Mihaela van der Schaar, University of California, Los Angeles (United States)

Anthony Vetro, Mitsubishi Electric Research Laboratories

(United States)

Session Chairs

Signal, Image, and Data Processing Plenary Session

Khan M. Iftekharuddin, Old Dominion University (United States) 
$1 \quad$ Medical Imaging

Peter Schelkens, Vrije Universiteit Brussel (Belgium)

2 Medical Imaging and Microscopy

Peter Schelkens, Vrije Universiteit Brussel (Belgium)

3 Remote Sensing and Space Imaging I

Yuriy A. Reznik, Brightcove, Inc. (United States)

4 Remote Sensing and Space Imaging II

Yuriy A. Reznik, Brightcove, Inc. (United States)

5 Next-Generation Image Compression

Thomas Richter, Fraunhofer-Institut für Integrierte Schaltungen (Germany)

6 Perceptual Coding, Quality Optimizations, and Assessments I

Dan Grois, Comcast Corporation (Israel)

7 Perceptual Coding, Quality Optimizations, and Assessments II

Dan Grois, Comcast Corporation (Israel)

8 Image Restoration and Enhancement I

Cristian Perra, Università degli Studi di Cagliari (Italy)

9 Future Video Coding I

Pankaj Topiwala, FastVDO Inc. (United States)

10 Future Video Coding II

Pankaj Topiwala, FastVDO Inc. (United States)

11 Light Field, Point Cloud, and Holographic Imaging I

Peter Schelkens, Vrije Universiteit Brussel (Belgium)

12 Light Field, Point Cloud, and Holographic Imaging II

Peter Schelkens, Vrije Universiteit Brussel (Belgium)

13 Image Restoration and Enhancement II

Thomas Richter, Fraunhofer-Institut für Integrierte Schaltungen (Germany)

14 Image Analysis and Security I

loannis Katsavounidis, Facebook Inc. (United States)

15 Image Analysis and Security II

loannis Katsavounidis, Facebook Inc. (United States) 